\title{
Infectious and Non-infectious Complications of Peritoneal Dialysis in Children
}

\section{Min Hyun Cho, M.D.}

Department of Pediatrics, Kyungpook National University, School of

Medicine, Daegu, South Korea

Corresponding author:

Min Hyun Cho, M.D.

Department of Pediatrics, Kyungpook

National University Hospital

130 Dongdeok-ro, Jung-gu, Daegu 41944,

Republic of Korea

Tel: +82-53-200-5719

Fax: +82-53-425-6683

E-mail: chomh@knu.ac.kr

Received: 22 July 2020

Revised: 10 August 2020

Accepted: 12 August 2020
Despite the many advantages of peritoneal dialysis (PD) in children with endstage renal disease, there exist redoubtable complications of PD that should be overcome. To prevent and manage these complications, a multidisciplinary team should provide support highly tailored for each child and family, based on the standardized practice guidelines for the management of pediatric PD. In this review, we summarize the clinical manifestations and management of several complications of PD.

Key words: Peritoneal dialysis, End-stage renal disease, Complication

\section{Introduction}

Peritoneal dialysis (PD) is the main modality of chronic renal replacement therapy in children, especially in infants and small children ${ }^{1)}$. PD has several advantages over hemodialysis including the ability to allow patients on it attend school on a regular basis, the absence of a need for regular intravascular access, better preservation of the residual renal function, and fewer dietary restrictions ${ }^{2}$. Despite the development of PD catheters and techniques, there are various complications of $\mathrm{PD}$ that should be overcome. Infectious complications of PD include exit-site infection (ESI), tunnel infection (TI), and peritonitis; while non-infectious complications include obstruction and malposition of PD catheters, fluid leaks, encapsulating peritoneal sclerosis (EPS), hernia, pain, and blood-stained dialysate. Per the International Society for Peritoneal Dialysis (ISPD) guidelines (2012 and 2016 updates) and other literature, this review summarizes the clinical manifestations and management of various complications of $\mathrm{PD}^{3,4)}$.

\section{Infectious complications}

under the terms of the Creative Commons Attribution Non-Commercial License (http:// creativecommons.org/licenses/by-nc/4.0/) which permits unrestricted non-commercial use, distribution, and reproduction in any medium, provided the original work is properly cited.

\section{Catheter-related infection: Exit-site infection (ESI) and tunnel infection (TI) \\ In the Standardizing Care to Improve Outcomes in Pediatric End-Stage Renal Disease (SCOPE) report, an annual rate of ESI was 0.25 per dialysis year and the median time taken to develop ESI was 392 days. In addition, perito-}


nitis occurred in $6 \%$ of cases, and the incidence of ESI was associated with age, being lowest in children $<2$ years ${ }^{5}$. To evaluate the status of the PD catheter exit site, the exit-site scoring system is recommended (Table 1), consisting of five parameters; exit-site swelling, crust, redness, pain on pressure, and secretion ${ }^{6}$. Each criterion is scored from 0 to 2 points ( 0 means no sign and 2 means definite finding). ESI should be assumed with a cumulative exit-site score of 4 or more. TI is defined by the presence of redness, edema, and tenderness along the subcutaneous portion of the catheter, with or without purulent drainage from the exit site $^{3)}$. In uncomplicated ESI, oral antibiotic therapy can be started and continued for a minimum of two weeks, and for at least 7 days after complete resolution of the infection. However, in cases of infection due to $S$. aureus or Pseudomonas, treatment for at least 3 weeks is recommended. In TI, the route of antibiotic administration can be oral, intraperitoneal, or intravenous; except for infection due to MRSA, which requires intravenous glycopeptide therapy, and treatment should last 2-4 weeks ${ }^{3)}$.

To decrease the incidence of ESI \& TI, the ISPD consensus guidelines recommend catheter immobilization for early optimal healing and once-weekly sterile dressing changes at the exit site, performed by experienced health personnel until the exit site is well healed ${ }^{3)}$. Cleansing the exit site with a sterile antiseptic solution and sterile gauze alongside the daily use of topical antibiotics (mupirocin or gentamicin) for chronic exit site care is also recommended 3,4).

\section{Peritonitis}

According to the data from the North American Pediatric Renal Trials and Collaborative Studies (NAPRTCS),

Table 1. Exit-site Scoring System*

\begin{tabular}{lccc}
\hline Indication & \multicolumn{3}{c}{ Score $^{\dagger}$} \\
\cline { 2 - 4 } Swelling & No & $\begin{array}{c}\text { Exit only } \\
(<0.5 \mathrm{~cm})\end{array}$ & $\begin{array}{c}\text { Including part of } \\
\text { or the entire tunnel }\end{array}$ \\
Crust & No & $<0.5 \mathrm{~cm}$ & $>0.5 \mathrm{~cm}$ \\
Redness & No & $<0.5 \mathrm{~cm}$ & $>0.5 \mathrm{~cm}$ \\
Pain on pressure & No & Slight & Severe \\
Secretion & No & Serous & Purulent
\end{tabular}

*From reference ${ }^{6}$.

${ }^{\dagger}$ A cumulative score according to five parameters of $\geq 4$ is considered indicative of an exit-site infection. the annualized rate of peritonitis in children was 0.68 (one episode every 17.8 months), and was inversely associated with age $(<1 \text { year, } 0.86 ;>12 \text { years, } 0.61)^{7}$. The incidence of peritonitis was also affected by the PD modality, with somewhat better rates for patients who were on automated PD as compared to those who were on continuous ambulatory $\mathrm{PD}^{7}$. Like in adults, the most common pathogens of peritonitis in children on PD are gram-positive organisms. About $50-60 \%$ of the peritonitis episodes are caused by gram-positive bacteria and $20-30 \%$ by gram-negative bacteria, with cultures remaining negative $(<20 \%)^{7}$. In the Korean pediatric report, $71.4 \%$ of peritonitis in children was caused by gram-positive bacteria and $73.9 \%$ developed during the first 6 months following initiation of $\mathrm{PD}^{8}$. In the SCOPE report, fungi were responsible for $8.0 \%$ of peritonitis episodes, predominantly occurring in children aged $<2$ years and associated with increased risk of hospitalization, longer hospital stay, and an increased frequency of technique failure ${ }^{9)}$. Furthermore, the upward orientation of the catheter exit site and touch contamination is associated with a higher risk of peritonitis ${ }^{10)}$.

To confirm the diagnosis of peritonitis, cloudy peritoneal effluent should be sent for cell count, differential count, and culture and if the white blood cell (WBC) count is greater than $100 / \mathrm{mm}^{3}$, and at least $50 \%$ of the WBCs are polymorphonuclear leukocytes, the peritonitis can be diagnosed empirically".

The ISPD consensus guidelines recommend that antibiotics for the treatment of bacterial peritonitis should be administered through the intraperitoneal route and continuous administration of beta-lactam antibiotics. For continuous therapy, the exchange with the loading dose should dwell for 3-6 hours; all subsequent exchanges during the treatment course should contain the maintenance dose. For intermittent therapy, the dose should be given once daily in the long-dwell ${ }^{3}$. In patients on nocturnal automated PD with short dwell times, prolongation of dwell time to 3-6 hours is required until the peritoneal effluent clears.

As the first empiric antibiotic therapy for peritonitis, intraperitoneal administration of a first-generation cephalosporin or vancomycin combined with a third-generation cephalosporin or an aminoglycoside is recommended. Cefepime has significant activity against gram-positive and 
gram-negative bacteria and monotherapy may be feasible ${ }^{4)}$. If the gram-positive bacteria are cultured and found to be susceptible to a first-generation cephalosporin, the thirdgeneration cephalosporin or an aminoglycoside can be discontinued. If the center-specific resistance rate of MRSA exceeds $10 \%$ or the patient has a history of MRSA, the replacement of a first-generation cephalosporin with a glycopeptide is recommended ${ }^{3,4)}$. Glycopeptide use can also be considered if the patient has a history of a severe allergy to penicillins and cephalosporins. In non-anuric patients receiving intermittent intraperitoneal doses of glycopeptide antibiotics (vancomycin or teicoplanin), they recommend monitoring the blood levels of the antibiotics ${ }^{3)}$. In addition, the ISPD consensus guidelines recommend adding rifampin in patients with $S$. aureus or coagulase-negative Staphylococcus who have a delayed response to initial therapy $(>72 \text { hours })^{3)}$. The duration of antibiotic therapy against gram-positive bacteria ranges from two to three weeks according to the organisms; $S$. aureus and enterococci: 21 days, coagulase-negative staphylococci, and other streptococci: 14 days.
If a gram-negative organism is cultured and found not to be Pseudomonas species, empiric glycopeptide therapy should be discontinued and either empiric cefepime or ceftazidime can be chosen as a single agent based on the susceptibility test. If Pseudomonas species is isolated, the addition of a second agent with a different mechanism of action should be considered. The duration of antibiotic therapy against gram-negative bacteria ranges from three to four weeks according to the organisms; Pseudomonas or Stenotrophomonas species: 21-28 days; other gram-negative bacilli: 21 days $^{3,4)}$.

If the initial cultures remain sterile at 72 hours and signs and symptoms of peritonitis are abated, empiric antibiotic therapy consisting of cefepime, ceftazidime, cefazolin, or a glycopeptide can be continued for two weeks ${ }^{3)}$ (Fig. 1).

In the case of refractory bacterial peritonitis, fungal peritonitis, ESI, or TI in conjunction with peritonitis with the same bacteria (except coagulase-negative staphylococcus), refractory ESI or TI, and repeated relapsing bacterial peritonitis, removal of the PD catheter should be considered ${ }^{3)}$. The ISPD consensus guidelines recommend a minimum

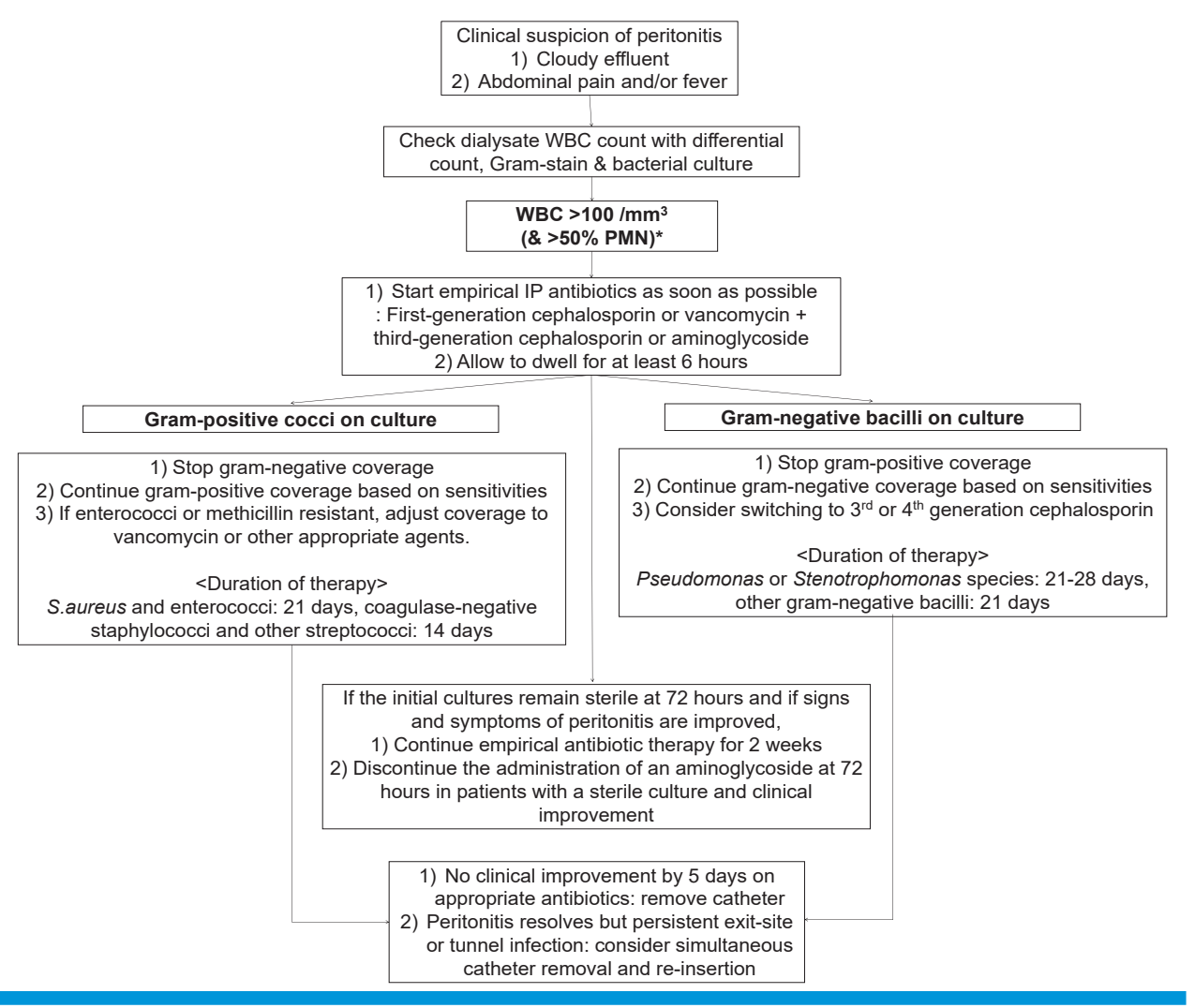

Fig. 1. Management algorithm for peritonitis. ${ }^{*} \mathrm{WBC}>100 / \mathrm{mm}^{3}$, of which at least $50 \%$ are PMN. WBC, white blood cell; PMN, polymorphonuclear neutrophils; IP, intraperitoneal. 
period of 2-3 weeks between catheter removal and insertion of a new catheter.

To decrease the incidence of peritonitis, the ISPD consensus guidelines recommend the use of a double-cuff Tenckhoff catheter with a downward or lateral subcutaneous tunnel configuration and perioperative antibiotic prophylaxis 60 minutes before the incision for PD catheter placement $t^{3)}$. For ostomy patients, it was recommended that gastrostomy placement should preferentially take place either before, or at the time of PD catheter placement. Also, to lower the risk, they suggest prophylactic administration of antibiotics after accidental intraluminal contamination and before invasive dental procedures and procedures involving the gastrointestinal or genitourinary tract ${ }^{3)}$.

\section{Non-infectious complications}

\section{Obstruction of the PD catheter}

In the report of Korean children on PD, the most common non-infectious complication was outflow failure (14.3 $\%)$, followed by fluid leaks and hernia ${ }^{11)}$. Causes of obstruction of PD catheters include wrapping of the omentum; entanglement of the ovary, fallopian tube, and small intestine; occlusion of the lumen by a blood clot; kinked tubing; and migration of the tube ${ }^{12}$. Obstruction rates of $\mathrm{PD}$ catheters between $12.5 \%$ and $36 \%$ have recently been reported in the pediatric population and children weighing less than $10 \mathrm{~kg}$ are more likely to develop this complication ${ }^{13)}$. The most common cause of obstruction was wrapping of the omentum around the PD catheter ${ }^{14)}$, as such prophylactic omentectomy during insertion of the PD catheter is recommended $^{12,15,16)}$.

PD catheter migration to undesired sites such as near the liver or spleen (Fig. 2) is one of the most frustrating complications resulting in dialysis failure and catheter removal ${ }^{17)}$. Inducing vigorous bowel peristalsis with laxatives or enemas may help in returning it. Bae et al. suggested that laparoscopic internal fixation of the PD catheter significantly decreases catheter migration rates without any additional complications ${ }^{17}$.

Recently, it was reported that $83 \%$ of PD access revisions were performed within the first year of PD treatment and the annual rate of access revision was 0.17 per treatment year ${ }^{2)}$. Major causes of access revisions included mechanical malfunction, peritonitis, ESI, and fluid leaks. The associated risk factors were younger age, diagnosis of congenital anomalies of the kidney and urinary tract, coexisting ostomies, and the presence of a swan-neck tunnel with a curled intraperitoneal portion ${ }^{2}$.

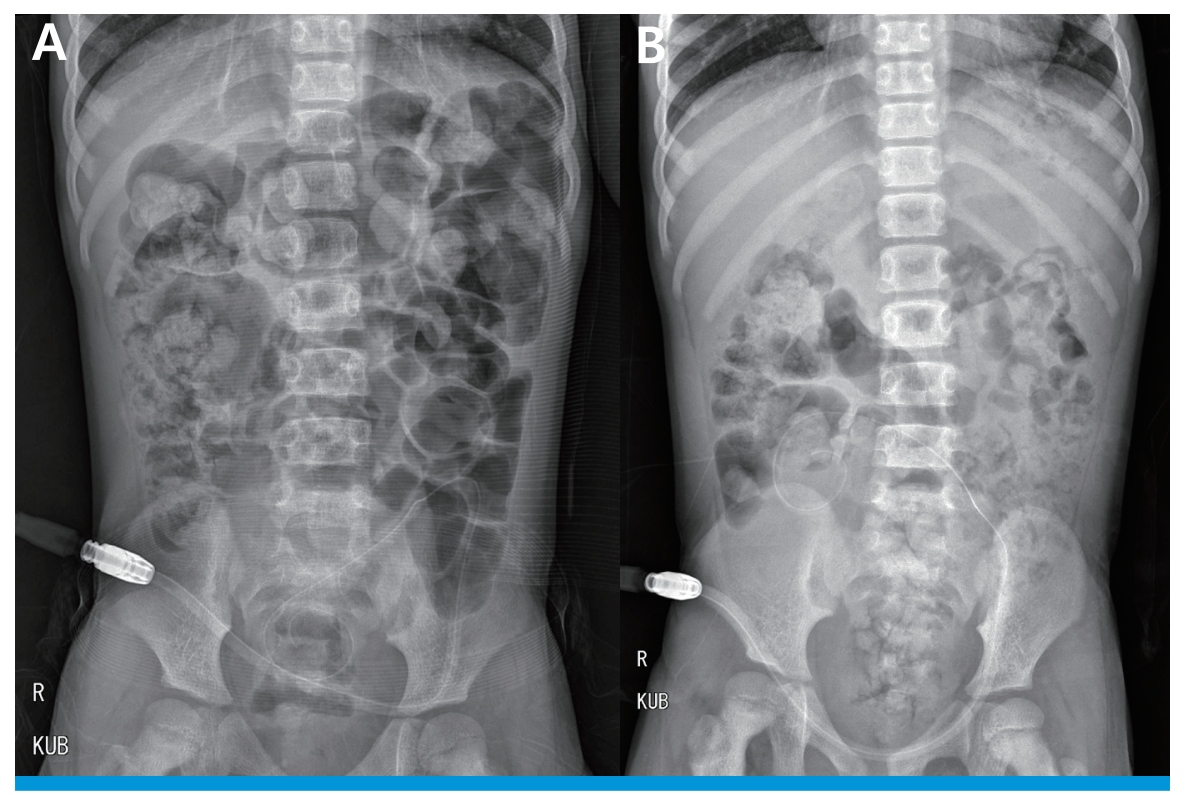

Fig. 2. Abdominal radiographs showing PD catheter migration developed in a 7-year-old girl. (A) Suitable position of the catheter tip just after the insertion of PD catheter (located in pelvic cavity). (B) Three days later, the tip of PD catheter migrated near liver and outflow failure developed. PD, peritoneal dialysis. 


\section{Fluid leaks}

Dialysate fluid leaks are one of the most frequent noninfectious complications of $\mathrm{PD}$, with pericatheter leaks being the most common ${ }^{12)}$. Fluid leaks can be developed in various areas, including higher volume leaks from a trocaremployed site, genital edema from an inguinal site, and extra-abdominal leaks such as hydrothorax and pericardial effusion ${ }^{12)}$. It is recommended that after PD catheter surgery, patients be allowed to heal sufficiently before use ( 2 weeks) to minimize this risk. General management of fluid leaks includes lowering the dialysate volume, performing PD with a cycler, and temporarily switching to hemodialysis, combined with surgical repair ${ }^{12}$.

\section{Encapsulating peritoneal sclerosis (EPS)}

EPS is a rare complication of PD associated with significant morbidity and mortality in adults ${ }^{18)}$. The ISPD definition of EPS is "a clinical syndrome continuously, intermittently, or repeatedly presenting with symptoms of intestinal obstruction due to adhesions of a diffusely thickened peritoneum" ${ }^{\text {19) }}$. Despite scarce pediatric data, the European Paediatric Dialysis Working Group (EPDWG) reported that the prevalence of EPS in children is comparable with that of adults, but mortality in children is significantly lower ${ }^{18)}$. According to the EPDWG survey, the prevalence of ESP in children was $1.5 \%$ (8.7 per 1,000 patient-years on PD) and these patients had a significantly higher peritonitis rate than non-EPS patients.

Several potential risk factors for developing EPS have been reported so far. First of all, long-term exposure to PD fluid is regarded as the most important risk factor for EPS. In the EPDWG survey, 68\% of EPS patients were on PD for $>5$ years ${ }^{18)}$. In the Japanese Registry study, the incidence of EPS was 6.6 and $12 \%$ in children on PD for $>5$ and $>8$ years, respectively ${ }^{20)}$. Recurrent episodes of peritonitis are known as another risk factor for developing EPS, particularly if the causative organism is Staphylococcus aureus, Enterococcus, Pseudomonas, or fungi ${ }^{211}$. Other potential risk factors include the use of acetate buffer, prolonged use of icodextrin, cessation of PD, and the use of chlorhexidine to sterilize tubing connections ${ }^{21)}$. Until now, despite no specific treatments of EPS, the general management of EPS includes termination of PD, the use of immunosuppressive and antifibrotic medicines, surgical intervention, and nutritional support.

\section{Hernia}

Hernias in the PD population are commonly incisional, umbilical, epigastric, or inguinal. The incidence of hernias in children on PD is inversely proportional to age. This may be explained by the more fragile abdominal wall and inguinal structures ${ }^{22}$. Since hernias tend to increase in size over time and are associated with an increased risk of bowel incarceration and strangulation, they generally require surgical repair, combined with lower volume cyclic $\mathrm{PD}$ as a temporary measure.

\section{Pain related to peritoneal dialysis}

After staring PD, some patients may have discomfort or pain during fluid inflow or outflow. Inflow pain occasionally affects the shoulders and is pleuritic in nature, which usually resolves over the following days. Slowing the rate of fluid inflow and the use of bicarbonate-lactate-buffered dialysate may improve symptoms in these patients ${ }^{23}$. Patients with outflow pain can be experienced in the genital area or rectum, as a result of pelvic irritation related to the tip of the catheter. The best treatment for outflow pain is leaving a small residual volume of fluid in the peritoneal cavity at the end of the drain, for example by using tidal automated PD.

\section{Bloody dialysate}

Bloody dialysate is uncommon and sometimes related to a history of abdominal trauma, ovulation, and menstruation. The treatment is to flush the abdomen with a few cycles of dialysate containing heparin (500 U/L) to minimize the chances of clotting in the catheter ${ }^{24)}$.

\section{Conclusions}

Although PD is the major modality of chronic renal replacement therapy and has several advantages over hemodialysis in children with end-stage renal disease, there are clinically significant complications that should be overcome. To prevent these complications, a standardized practice guideline for the management of pediatric $\mathrm{PD}$ should be updated and dissipated. Also, a multidisciplinary 
team consisting of pediatric nephrologists, pediatric surgeons, and PD nurses should provide support tailored to each child and family.

\section{Conflicts of interest}

\author{
No potential conflict of interest relevant to this article \\ was reported.
}

\section{ORCID ID}

\section{Min Hyun Cho https://orcid.org/0000-0002-7965-7587}

\section{References}

1. Woodrow G, Fan SL, Reid C, Denning J, Pyrah AN. Renal Association Clinical Practice Guideline on peritoneal dialysis in adults and children. BMC Nephrol 2017;18:333.

2. Borzych-Duzalka D, Aki TF, Azocar M, White C, Harvey E, Mir S, et al. Peritoneal Dialysis Access Revision in Children: Causes, Interventions, and Outcomes. Clin J Am Soc Nephrol 2017;12:105-12.

3. Warady BA, Bakkaloglu S, Newland J, Cantwell M, Verrina E, Neu $A$, et al. Consensus guidelines for the prevention and treatment of catheter-related infections and peritonitis in pediatric patients receiving peritoneal dialysis: 2012 update. Perit Dial Int 2012;32 Suppl 2:S32-86.

4. Li PK, Szeto CC, Piraino B, de Arteaga J, Fan S, Figueiredo AE, et al. ISPD Peritonitis Recommendations: 2016 Update on Prevention and Treatment. Perit Dial Int 2016:36:481-508.

5. Swartz SJ, Neu A, Skversky Mason A, Richardson T, Rodean J, Lawlor J, et al. Exit site and tunnel infections in children on chronic peritoneal dialysis: findings from the Standardizing Care to Improve Outcomes in Pediatric End Stage Renal Disease (SCOPE) Collaborative. Pediatr Nephrol 2018;33:1029-35.

6. Schaefer F, Klaus G, Müller-Wiefel DE, Mehls O. Intermittent versus continuous intraperitoneal glycopeptide/ceftazidime treatment in children with peritoneal dialysis-associated peritonitis. The Mid-European Pediatric Peritoneal Dialysis Study Group (MEPPS). J Am Soc Nephrol 1999;10:136-45.

7. Chadha V, Schaefer FS, Warady BA. Dialysis-associated peritonitis in children. Pediatr Nephrol 2010;25:425-40.

8. Lee KO, Park SJ, Kim JH, Lee JS, Kim PK, Shin Jl. Outcomes of peritonitis in children on peritoneal dialysis: a 25-year experience at Severance Hospital. Yonsei Med J 2013;54:983-9.

9. Munshi R, Sethna CB, Richardson T, Rodean J, Al-Akash S, Gupta $S$, et al. Fungal peritonitis in the Standardizing Care to Improve
Outcomes in Pediatric End Stage Renal Disease (SCOPE) Collaborative. Pediatr Nephrol 2018;33:873-80.

10. Sethna CB, Bryant K, Munshi R, Warady BA, Richardson T, Lawlor J, et al. Risk Factors for and Outcomes of Catheter-Associated Peritonitis in Children: The SCOPE Collaborative. Clin J Am Soc Nephrol 2016;11:1590-6.

11. Kim JE, Park SJ, Oh JY, Kim JH, Lee JS, Kim PK, et al. Noninfectious Complications of Peritoneal Dialysis in Korean Children: A 26-Year Single-Center Study. Yonsei Med J 2015;56:1359-64.

12. Fraser N, Hussain FK, Connell R, Shenoy MU. Chronic peritoneal dialysis in children. Int J Nephrol Renovasc Dis 2015;8:125-37.

13. Radtke J, Lemke A, Kemper MJ, Nashan B, Koch M. Surgical complications after peritoneal dialysis catheter implantation depend on children's weight. J Pediatr Surg 2016;51:1317-20.

14. Lemoine C, Keswani M, Superina R. Factors associated with early peritoneal dialysis catheter malfunction. J Pediatr Surg 2019;54: 1069-75.

15. Phan J, Stanford S, Zaritsky JJ, DeUgarte DA. Risk factors for morbidity and mortality in pediatric patients with peritoneal dialysis catheters. J Pediatr Surg 2013;48:197-202.

16. Cribbs RK, Greenbaum LA, Heiss KF. Risk factors for early peritoneal dialysis catheter failure in children. J Pediatr Surg 2010;45: 585-9.

17. Bae IE, Chung WK, Choi ST, Kang J. Laparoscopic internal fixation is a viable alternative option for continuous ambulatory peritoneal dialysis catheter insertion. J Korean Surg Soc 2012;83:381-7.

18. Shroff R, Stefanidis CJ, Askiti V, Edefonti A, Testa S, Ekim M, et al. Encapsulating peritoneal sclerosis in children on chronic PD: a survey from the European Paediatric Dialysis Working Group. Nephrol Dial Transplant 2013;28:1908-14.

19. Kawaguchi Y, Kawanishi H, Mujais S, Topley N, Oreopoulos DG. Encapsulating peritoneal sclerosis: definition, etiology, diagnosis, and treatment. International Society for Peritoneal Dialysis Ad Hoc Committee on ultrafiltration management in peritoneal dialysis. Perit Dial Int 2000;20(Suppl 4):S43-S55.

20. Hoshii S, Honda M, Itami N, Oh S, Matsumura C, Moriya S, et al. Sclerosing encapsulating peritonitis in pediatric peritoneal dialysis patients. Pediatr Nephrol 2000;14:275-9.

21. Stefanidis CJ, Shroff R. Encapsulating peritoneal sclerosis in children. Pediatr Nephrol 2014;29:2093-103.

22. van AsseldonkJP, Schröder CH, Severijnen RS, de Jong MC, Monnens LA. Infectious and surgical complications of childhood continuous ambulatory peritoneal dialysis. Eur J Pediatr 1992;151: 377-80.

23. Mactier RA, Sprosen TS, Gokal R, Williams PF, Lindbergh M, Naik $\mathrm{RB}$, et al. Bicarbonate and bicarbonate/lactate peritoneal dialysis solutions for the treatment of infusion pain. Kidney Int 1998; 531061-7.

24. Lew SQ. Hemoperitoneum: bloody peritoneal dialysate in ESRD patients receiving peritoneal dialysis. Perit Dial Int 2007;27:22633. 